\title{
Relationships between lipid profiles and metabolic syndrome, insulin resistance and serum high molecular adiponectin in Japanese community-dwelling adults
}

Ryuichi Kawamoto ${ }^{1,3^{*}}$, Yasuharu Tabara ${ }^{2}$, Katsuhiko Kohara ${ }^{2}$, Tetsuro Miki ${ }^{2}$, Tomo Kusunoki ${ }^{1,3}$, Shuzo Takayama ${ }^{1}$, Masanori Abe', Tateaki Katoh ${ }^{3}$ and Nobuyuki Ohtsuka ${ }^{3}$

\begin{abstract}
Background: There are few studies to demonstrate the associations between newly addressed lipid profiles and metabolic syndrome (MetS)-associated variables.

Methods: Study participants without medications for hypertension, diabetes, or dyslipidemia $\{614$ men aged $58 \pm$ 14 (mean \pm standard deviation; range, 20-89) years and 779 women aged $60 \pm 12$ (range, 21-88) years\} were randomly recruited from a single community at the time of their annual health examination. The association between lipid profiles (total cholesterol (T-C), triglycerides (TG), low-density lipoprotein cholesterol (LDL-C), highdensity lipoprotein cholesterol (HDL-C), non-HDL-C, T-C/HDL-C, TG/HDL-C, LDL-C/HDL-C ratio and MetS, Insulin resistance by homeostasis model assessment of insulin resistance (HOMA-IR), and serum HMW adiponectin were analyzed.

Results: In multiple linear regression analysis, TG/HDL-C and T-C/HDL-C ratios as well as TG showed significantly strong associations with all three MetS-associated variables in both men and women. In men, the ROC curve analyses showed that the best marker for these variables was TG/HDL-C ratio, with the AUC for presence of MetS (AUC, 0.82; 95\% Cl, 0.77-0.87), HOMA-IR (AUC, 0.75; 95\% Cl, 0.70-0.80), and serum HMW adiponectin (AUC, 0.67; 95\% $\mathrm{Cl}, 0.63-0.71)$, respectively. The T-C/HDL-C ratio, TG, HDL-C, LDL-C/HDL-C ratio, and non-HDL-C also discriminated these markers; however all their AUC estimates were lower than TG/HDL-C ratio. These results were similar in women.

Conclusion: In Japanese community-dwelling adults, lipid ratios of TG/HDL-C, T-C/HDL-C, LDL-C/HDL-C as well as TG and HDL-C were consistently associated with MetS, insulin resistance and serum HMW adiponectin. Lipid ratios may be used as reliable markers.
\end{abstract}

\section{Background}

Metabolic syndrome (MetS) known as a clustering of cardiovascular risk factors associated with insulin resistance, hypertension, glucose intolerance, hypertriglyceridemia $[1,2]$, and low levels of high-density lipoprotein cholesterol (HDL-C), is a major worldwide public health problem. In Japan also, MetS is quite common, affecting

\footnotetext{
* Correspondence: rykawamo@yahoo.co.jp

'Department of Community Medicine, Ehime University Graduate School of Medicine, Ehime 791-0295, Japan

Full list of author information is available at the end of the article
}

$13.3 \%$ to $25.3 \%$ of Japanese men [3,4] and may become even more common in the future with the continuous increase in the prevalence of obesity. Several epidemiological studies have demonstrated that MetS increases the risk of various cardiovascular diseases (CVD) [5] and diabetes [6].

Serum adiponectin, which is a 247 -amino acid protein secreted specifically by adipose tissue, contains four differentiable domains that regulate lipid metabolism, glucose metabolism, and insulin sensitivity [7], and low circulating levels of serum adiponectin has been

\section{Ciomed Central}


reported as a risk factor for the development of metabolic syndrome [8] and CVD [9]. The high molecular weight (HMW) form binds most avidly to its receptors and is one of the important molecules activating metabolic role of adiponectin [10].

Dyslipidemia is another well-known risk factor for CVD, as well as a component of MetS, and the role of HDL-C, triglycerides (TG) and low-density lipoprotein cholesterol (LDL-C) are already established as predictors of CVD [11]. On the other hand, a characteristic dyslipidemia is also associated with insulin resistance [12]. Several studies have reported the possibility that newly addressed lipid profiles might be more useful than the traditional ones used for CVD prediction, and measuring these variables might help identify insulin resistance and CVD [13]. Total cholesterol (T-C)/HDL-C, TG/ HDL-C, and LDL-C/HDL-C ratio [13-15], as well as TG and HDL-C [16] are independently associated with insulin resistance and risk factors of CVD. However, in Japanese community-dwelling persons, there are few studies to demonstrate the associations between these lipid profiles, especially the lipid ratios, with MetS, insulin resistance and serum HMW adiponectin.

We took advantage of the large representative sample of Japanese adults who participated at the time of their annual health examination. We investigated how lipid profiles were associated with MetS, insulin resistance and serum HMW adiponectin in healthy Japanese adults. For this, we used cross-sectional data from community-dwelling participants without medication and clinical diabetes.

\section{Methods \\ Subjects}

Participants were recruited at the time of their annual health examination. The sample population compromised 3,164 middle-aged to elderly residents. Information on medical history, present conditions, and drugs were obtained by interview. Other characteristics, e.g., smoking and alcohol habits, and medication, were investigated by individual interviews using a structured questionnaire. Subjects taking medications for hypertension, diabetes, or dyslipidemia were excluded. The final study sample included 614 men and 779 women. This study was approved by the ethics committee of Ehime University School of Medicine, and written informed consent was obtained from each subject.

\section{Evaluation of confounding factors}

Information on demographic characteristics and risk factors was collected using clinical files. Body mass index (BMI) was calculated by dividing weight (in kilograms) by the square of the height (in meters). We measured blood pressure in the right upper arm of participants in a sedentary position using an automatic oscillometric blood pressure recorder (BP-103i; Colin, Aichi, Japan) while the subjects were seated after having rested for at least $5 \mathrm{~min}$. Smoking status was defined as the number of cigarette packs per day multiplied by the number of years smoked (pack - year), and the participants were classified into never smokers, past smokers, light smokers $(<30$ pack - year) and heavy smokers ( $\geq 30$ pack · year). The daily alcohol consumption was measured using the Japanese liquor unit in which a unit corresponds to $22.9 \mathrm{~g}$ of ethanol, and the participants were classified into never drinkers, occasional drinkers ( $<1$ unit/day), light drinkers (1-1.9 unit/day), and heavy drinkers ( $\geq 2$ unit/day). T-C, TG, HDL-C, fasting blood glucose (FBG), creatinine (enzymatic method), immunoreactive insulin (IRI), and serum HMW adiponectin (FUJIREBIO, Tokyo, Japan) were measured during fasting. LDL-C levels were calculated using the Friedewald formula [17]. Participants with TG levels $\geq 400 \mathrm{mg} / \mathrm{dl}$ were excluded. Homeostasis model assessment of insulin resistance (HOMA-IR) was calculated from FBG and IRI levels using the following formula; $\{$ FBG $(\mathrm{mg} / \mathrm{dL}) \times \mathrm{IRI}(\mathrm{mU} / \mathrm{mL})\} / 405$ [18].

\section{Metabolic Syndrome}

We applied condition-specific cutoff points for MetS based on the modified guidelines for the diagnosis of MetS in Japan [19]. Metabolic syndrome was defined as obesity with at least two of the following three conditions: hypertension, dyslipidemia, and impaired fasting glucose (IFG). Obesity was defined as a BMI of $\geq 25.0$ $\mathrm{kg} / \mathrm{m}^{2}$ [20]. Hypertension was defined as systolic blood pressure $(\mathrm{SBP}) \geq 130 \mathrm{mmHg}$ and diastolic blood pressure (DBP) $\geq 85 \mathrm{mmHg}$. Dyslipidemia was defined as TG concentrations $\geq 150 \mathrm{mg} / \mathrm{dL}$ and low HDL cholesterolemia (HDL-C $<40 \mathrm{mg} / \mathrm{dL}$ ). IFG was defined as a FBG level $\geq 110 \mathrm{mg} / \mathrm{dL}$.

\section{Statistical analysis}

Statistical analysis was performed using PASW Statistics 17.0 (Statistical Package for Social Science Japan, Inc., Tokyo, Japan). All values are expressed as mean \pm standard deviation (SD), unless otherwise specified. Data for TG, FBG, HOMA-IR, and serum HMW adiponectin were skewed, and log-transformed for analysis. Differences between the two groups were determined by Student's t-test and $\chi^{2}$ test. Subjects were divided into four groups based on the quartile of HOMA-IR and serum HMW adiponectin levels and the cutoff points for metabolic disorder were defined as the fourth quartile of HOMA-IR and first quartile of serum HMW adiponectin levels, respectively. Multiple linear regression analysis was used to evaluate the contribution of each lipid 
profile for the number of MetS components, HOMA-IR, and serum HMW adiponectin. In addition, areas under the receiver operating characteristic (ROC) curves were determined for each variable to identify the predictors of MetS-associated variables. The area under the curve (AUC) of ROC curves is a summary of the overall diagnostic accuracy of the test. The best markers have ROC curves that are shifted to the left with areas under the curve near unity. Nondiagnostic markers are represented by diagonals with the AUC of ROC curves close to 0.5. A value of $P<0.05$ was considered significant.

\section{Results}

\section{Background factors of subjects categorized by sex}

Table 1 shows the value of each background factor categorized by sex. The subjects comprised 614 men aged $58 \pm 14$ (range, 20-89) years and 779 women aged $60 \pm$ 12 (range, 21-88) years. The mean BMI was $23.3 \pm 3.0$ $\mathrm{kg} / \mathrm{m}^{2}$ and $22.9 \pm 3.2 \mathrm{~kg} / \mathrm{m}^{2}$ in men and women, respectively. BMI, smoking status, alcohol consumption, systolic blood pressure (SBP), diastolic blood pressure (DBP), $\mathrm{TG}$, and TG/HDL-C ratio were significantly higher but age, T-C, HDL-C, LDL-C, and Non-HDL-C were significantly lower in men than in women. There were no inter-group differences regarding $\mathrm{T}-\mathrm{C} / \mathrm{HDL}-\mathrm{C}$ ratio, LDL-C/HDL-C ratio, eGFR, and prevalence of CVD.

\section{Insulin resistance of subjects categorized by sex}

Prevalence of MetS and FBG were significantly higher in men than in women, but IRI, HOMA-IR and serum HMW adiponectin were significantly lower in men (Table 2).
Table 2 Insulin resistance and prevalence of metabolic syndrome of subjects categorized by sex

\begin{tabular}{|c|c|c|c|}
\hline Characteristics & $\begin{array}{c}\text { Men } \\
\mathrm{N}=614\end{array}$ & $\begin{array}{l}\text { Women } \\
\mathrm{N}=779\end{array}$ & $\begin{array}{c}P \\
\text {-value* }\end{array}$ \\
\hline Metabolic syndrome, \% & 14.2 & 8.3 & 0.001 \\
\hline $\begin{array}{l}\text { Fasting blood glucose } \\
(\mathrm{mg} / \mathrm{dL})\end{array}$ & $94(89-101)$ & $91(86-97)$ & $<0.001$ \\
\hline $\begin{array}{l}\text { Immuno-reactive insulin } \\
(\mathrm{mU} / \mathrm{mL})\end{array}$ & $4.4(2.8-7.2)$ & $5.6(3.8-8.0)$ & $<0.001$ \\
\hline HOMA-IR§ & $1.05(0.64-1.81)$ & $1.27(0.84-1.84)$ & $<0.001$ \\
\hline Serum HMW adiponectin & $3.20(1.97-4.92)$ & $6.65(4.42-9.64)$ & $<0.001$ \\
\hline
\end{tabular}
$(\mu \mathrm{g} / \mathrm{mL})$

HOMA-IR, homeostasis model assessment of insulin resistance; HMW, high molecular weight. Data for fasting blood glucose, fasting insulin, HOMA-IR, and serum HMW adiponectin were skewed and presented as median (interquartile range) values. §HOMA-IR was calculated using the following formula; \{fasting blood glucose (FBG) $(\mathrm{mg} / \mathrm{dL}) \mathrm{X}$ fasting insulin $(\mathrm{mU} / \mathrm{mL})\} / 405$. Data for fasting blood glucose, immuno-reactive insulin, HOMA-IR, and serum HMW adiponectin were log-transformed for analysis. * Student's t-test or $\chi^{2}$ test.

\section{Synergistic effect of increased HOMA-IR and reduced serum HMW adiponectin}

Figure 1 showed synergistic effect of increased HOMAIR and reduced serum HMW adiponectin. Mean accumulated number of MetS components was the highest in the highest HOMA-IR-lowest serum HMW adiponectin quartile group and lowest in the lowest HOMA-IRhighest serum HMW adiponectin quartile group, while HOMA-IR appears to be a more dominant determinant for MetS components than serum HMW adiponectin. HOMA-IR and serum HMW adiponectin were independently associated with the number of MetS components. We assessed the statistical significance of the relationship using a general linear model with the following

Table 1 Characteristics of subjects categorized by sex

\begin{tabular}{|c|c|c|c|}
\hline Characteristics & Men N = 614 & Women $\mathrm{N}=779$ & $P$-value* \\
\hline Age (years) & $58 \pm 14$ & $60 \pm 12$ & 0.001 \\
\hline Body mass index $\left(\mathrm{kg} / \mathrm{m}^{2}\right)$ & $23.3 \pm 3.0$ & $22.9 \pm 3.2$ & 0.027 \\
\hline Smoking status \{never/ex/light/heavy (\%)\} & $37.8 / 20.8 / 17.9 / 23.5$ & $97.2 / 0.8 / 1.9 / 0.1$ & $<0.001$ \\
\hline Alcohol consumption \{never/light/moderate/heavy (\%)) & $12.2 / 29.5 / 34.7 / 23.6$ & $60.1 / 33.4 / 5.9 / 0.6$ & $<0.001$ \\
\hline Systolic blood pressure (mmHg) & $135 \pm 19$ & $132 \pm 22$ & 0.005 \\
\hline Diastolic blood pressure $(\mathrm{mmHg})$ & $82 \pm 11$ & $78 \pm 12$ & $<0.001$ \\
\hline Total cholesterol (mg/dL) & $191 \pm 34$ & $209 \pm 33$ & $<0.001$ \\
\hline Triglycerides (mg/dL) & $97(71-140)$ & $85(63-115)$ & $<0.001$ \\
\hline HDL cholesterol (mg/dL) & $58 \pm 14$ & $65 \pm 15$ & $<0.001$ \\
\hline LDL cholesterol (mg/dL) & $109 \pm 32$ & $124 \pm 30$ & $<0.001$ \\
\hline Non-HDL cholesterol (mg/dL) & $132 \pm 34$ & $143 \pm 33$ & $<0.001$ \\
\hline Total cholesterol/HDL cholesterol ratio & $3.45 \pm 1.01$ & $3.35 \pm 0.92$ & 0.066 \\
\hline Triglycerides/HDL cholesterol ratio & $1.67(1.16-2.74)$ & $1.29(0.89-1.97)$ & 0.001 \\
\hline LDL cholesterol/HDL cholesterol ratio & $2.00 \pm 0.81$ & $2.02 \pm 0.75$ & 0.601 \\
\hline eGFR & $83.9 \pm 16.3$ & $82.7 \pm 16.5$ & 0.183 \\
\hline Cardiovascular disease, \% & 5.7 & 3.9 & 0.124 \\
\hline
\end{tabular}

$\mathrm{HDL}$, high-density lipoprotein; LDL, low-density lipoprotein; eGFR, estimated glomerular filtration rate. Data presented are mean \pm standard deviation. Data for triglycerides and triglycerides/HDL cholesterol ratio were skewed and were presented as median (interquartile range). Data for triglycerides and triglycerides/HDL cholesterol ratio were log-transformed for analysis. * Student's t-test or $\chi^{2}$ test. 


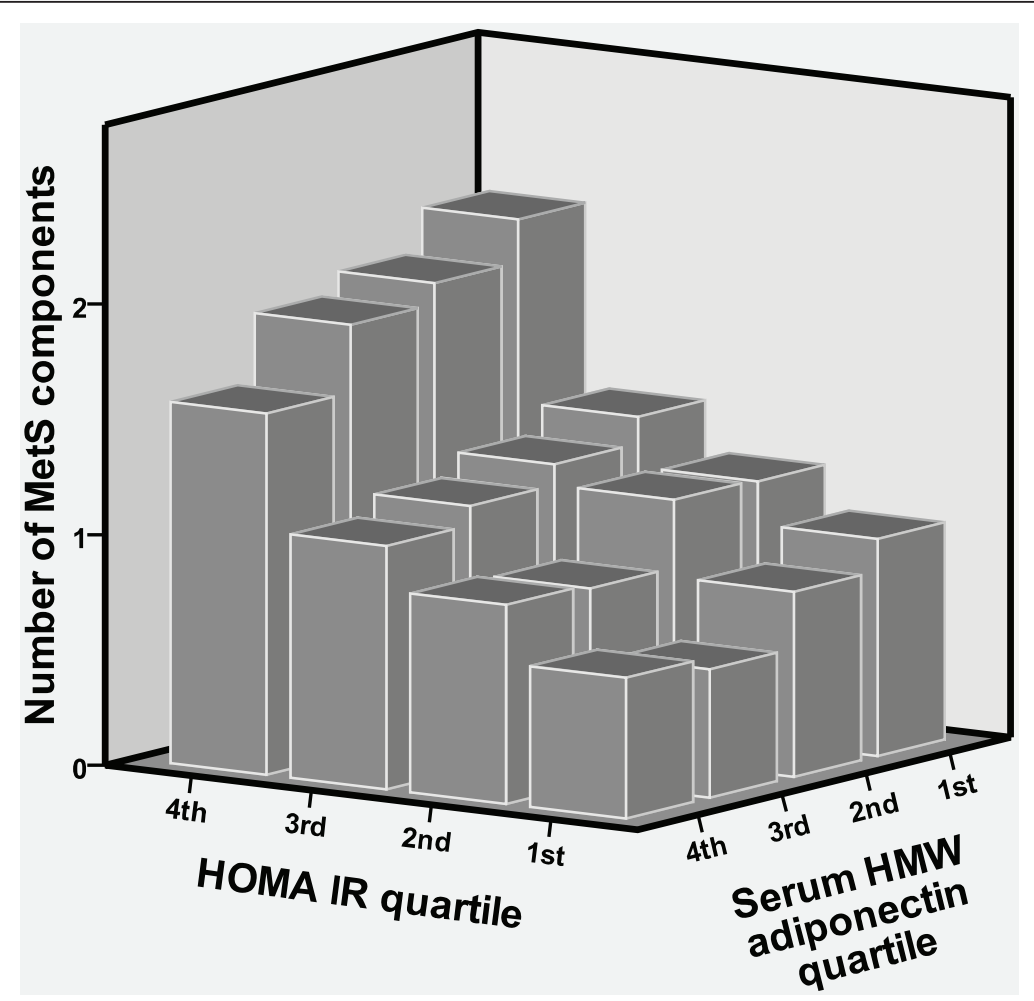

Figure 1 Synergistic effect of increased HOMA-IR (homeostasis model assessment of insulin resistance) and reduced serum HMW (high molecular weight) adiponectin. Mean accumulated number of the following MetS (metabolic syndrome) components: obesity, increased Blood pressure, impaired fasting glucose, dyslipidemia (hypertriglyceridemia or low high-density lipoprotein cholesterolemia). Study participants were divided into four groups (quartile) according to HOMA-IR and serum HMW adiponectin levels. Each quartile was calculated within sexes and then combined to eliminate any gender differences. Statistical significance was assessed by a general linear model for the following confounding factors: age, sex, BMI, smoking status, alcohol consumption, SBP, DBP, and eGFR.

confounding factors: age, sex, BMI, smoking status, alcohol consumption, SBP, DBP, and eGFR. The interaction between increased HOMA-IR and reduced serum HMW adiponectin was a significant and dependent determinant for the accumulated number of MetS components $(\mathrm{F}=18.7, P<0.001)$, in addition to their direct association (HOMA-IR, $\mathrm{F}=77.0, P<0.001$; serum HMW adiponectin, $\mathrm{F}=9.71, P=0.002$ ).

\section{Relationship between various risk factors including lipid} profiles and MetS-associated variables categorized by sex To further investigate whether lipid profiles can explain the MetS-associated variables independent of other known confounding factors, multiple linear regression analyses for the number of MetS components, HOMAIR, and serum HMW adiponectin were performed (Table 3). In both men and women, TG/HDL-C and T$\mathrm{C} / \mathrm{HDL}-\mathrm{C}$ ratios as well as TG showed significantly strong associations with all 3 of the MetS-associated variables. Non-HDL-C and LDL-C/HDL-C ratio also showed significant correlations; however almost their $\beta$ estimates were lower than the TG/HDL-C ratio.
Comparison of areas under ROC curves $(95 \% \mathrm{Cl})$ for potential markers of MetS-associated variables of subjects categorized by sex

In men, the ROC curve analyses showed that the best marker of the MetS-associated variables (Presence of MetS, HOMA-IR $>1.98$, and serum HMW adiponectin $<2.97 \mu \mathrm{g} / \mathrm{mL}$ ) was TG/HDL-C ratio, with the AUC for presence of MetS (AUC, 0.82; 95\% CI, 0.77-0.87), HOMA-IR (AUC, 0.75; 95\% CI, 0.70-0.80), and serum HMW adiponectin (AUC, 0.67; 95\% CI, 0.63-0.71), respectively (Table 4 ). The T-C/HDL-C ratio, TG, HDL$\mathrm{C}$, non-HDL-C, and LDL-C/HDL-C ratio also discriminated these markers; however all their AUC estimates were lower than the TG/HDL-C ratio. These results were similar in women. On the other hand, each AUC of LDL-C for these three variables was near 0.50 in both women and men.

\section{Discussion}

In the present study, we examined whether lipid profiles (i.e., TG, HDL-C, LDL-C, non-HDL-C, T-C/HDL-C ratio, TG/HDL-C ratio, and LDL-C/HDL-C ratio) were 
Table 3 Multiple linear regression analysis of the lipid measures with the number of MetS components, HOMA-IR and serum HMW adiponectin of subjects categorized by sex

\begin{tabular}{|c|c|c|c|c|c|c|}
\hline \multirow[t]{4}{*}{ Characteristics } & \multicolumn{6}{|c|}{$\beta$ (P-value) } \\
\hline & \multicolumn{2}{|c|}{ Number. of MetS components } & \multicolumn{2}{|c|}{ HOMA-IR§ } & \multicolumn{2}{|c|}{ Serum HMW adiponectin } \\
\hline & Men & Women & Men & Women & Men & Women \\
\hline & $N=614$ & $\mathrm{~N}=779$ & $N=614$ & $\mathrm{~N}=779$ & $N=614$ & $\mathrm{~N}=779$ \\
\hline Triglycerides (mg/dL) & $0.337(<0.001)$ & $0.266(<0.001)$ & $0.237(<0.001)$ & $0.231(<0.001)$ & $-0.232(<0.001)$ & $-0.237(<0.001)$ \\
\hline HDL cholesterol (mg/dL) & $-0.193(<0.001)$ & $-0.121(<0.001)$ & $-0.175(<0.001)$ & $-0.122(<0.001)$ & $0.218(<0.001)$ & $0.329(<0.001)$ \\
\hline LDL cholesterol (mg/dL) & $-0.021(0.503)$ & $0.051(0.048)$ & $0.091(0.009)$ & $0.107(0.001)$ & $-0.126(0.001)$ & $-0.176(0.001)$ \\
\hline Non-HDL cholesterol (mg/dL) & $0.115(<0.001)$ & $0.134(<0.001)$ & $0.164(<0.001)$ & $0.158(<0.001)$ & $-0.188(<0.001)$ & $-0.227(<0.001)$ \\
\hline Total cholesterol/HDL cholesterol ratio & $0.256(<0.001)$ & $0.201(<0.001)$ & $0.252(<0.001)$ & $0.198(<0.001)$ & $-0.295(<0.001)$ & $-0.362(<0.001)$ \\
\hline Triglycerides/HDL cholesterol ratio & $0.354(<0.001)$ & $0.257(<0.001)$ & $0.258(<0.001)$ & $0.228(<0.001)$ & $-0.270(<0.001)$ & $-0.315(<0.001)$ \\
\hline LDL cholestero//HDL cholesterol ratio & $0.143(<0.001)$ & $0.148(<0.001)$ & $0.209(<0.001)$ & $0.174(<0.001)$ & $-0.265(<0.001)$ & $-0.352(<0.001)$ \\
\hline
\end{tabular}

MetS, Metabolic syndrome; HOMA-IR, homeostasis model assessment of insulin resistance; HMW, high molecular weight; HDL, high-density lipoprotein; LDL, lowdensity lipoprotein. §HOMA-IR was calculated using the following formula; \{fasting blood glucose (mg/dL) X immuno-reactive insulin (mU/mL)\}/405. Adjusted for age were BMI, smoking status, alcohol consumption, SBP, DBP, and eGFR. Data for triglycerides, triglycerides/HDL cholesterol ratio, and HOMA-IR were skewed, and log-transformed for analysis.

associated with MetS-associated variables (i.e., MetS, HOMA-IR, and serum HMW adiponectin) in Japanese community-dwelling adults. We demonstrated that HOMA-IR and serum HMW adiponectin, an active form of adiponectin, synergistically reflected the accumulated number of MetS components, and TG/HDL-C and $\mathrm{T}-\mathrm{C} / \mathrm{HDL}-\mathrm{C}$ ratios, moreover, TG showed significantly and independently strong associations with these MetS-associated variables in both men and women. The best marker of three MetS-associated variables was TG/ HDL-C ratio.
Resistance to insulin-mediated glucose disposal is distributed continuously through the general population [21], but we have no measurement which identify a participant with insulin resistance or insulin sensitive. Direct measurement of insulin resistance using the hyperinsulinemic-euglycemic clamp has practical limitations [22]. Some previous studies have shown that HOMA-IR based insulin resistance measurements have a strong correlation with glucose clamp-assessed insulin resistance $[18,21]$. Although these methods are less accurate than the hyperinsulinemic-euglycemic clamp,

Table 4 Comparison of areas under the ROC curves $(95 \% \mathrm{Cl})$ for potential markers of insulin resistance of subjects categorized by sex

\begin{tabular}{|c|c|c|c|c|c|c|}
\hline \multirow[b]{2}{*}{ Characteristics } & \multicolumn{2}{|c|}{ Presence of MetS } & \multicolumn{2}{|c|}{ HOMA-IR§ >1.98 } & \multicolumn{2}{|c|}{ Serum HMW adiponectin $<2.97 \mu \mathrm{g} / \mathrm{mL}$} \\
\hline & AUC $(95 \% \mathrm{Cl})$ & $P$-value & $\operatorname{AUC}(95 \% \mathrm{Cl})$ & $P$-value & $\operatorname{AUC}(95 \% \mathrm{Cl})$ & $P$-value \\
\hline \multicolumn{7}{|l|}{ Men, $N=614$} \\
\hline Triglycerides (mg/dL) & $0.81(0.76-0.86)$ & $<0.001$ & $0.73(0.68-0.78)$ & $<0.001$ & $0.67(0.63-0.71)$ & $<0.001$ \\
\hline HDL cholesterol (mg/dL) & $0.28(0.22-0.34)$ & $<0.001$ & $0.31(0.26-0.36)$ & $<0.001$ & $0.40(0.35-0.44)$ & $<0.001$ \\
\hline LDL cholesterol (mg/dL) & $0.56(0.50-0.63)$ & $<0.001$ & $0.60(0.54-0.65)$ & $<0.001$ & $0.51(0.47-0.56)$ & 0.577 \\
\hline Non-HDL cholesterol (mg/dL) & $0.68(0.62-0.74)$ & $<0.001$ & $0.66(0.61-0.71)$ & $<0.001$ & $0.56(0.51-0.61)$ & 0.011 \\
\hline Total cholesterol/HDL cholesterol ratio & $0.76(0.70-0.81)$ & $<0.001$ & $0.73(0.68-0.78)$ & $<0.001$ & $0.61(0.56-0.65)$ & $<0.001$ \\
\hline Triglycerides/HDL cholesterol ratio & $0.82(0.77-0.87)$ & $<0.001$ & $0.75(0.70-0.80)$ & $<0.001$ & $0.67(0.63-0.71)$ & $<0.001$ \\
\hline LDL-cholesterol/HDL-cholesterol ratio & $0.68(0.62-0.74)$ & $<0.001$ & $0.69(0.63-0.74)$ & $<0.001$ & $0.57(0.53-0.62)$ & 0.002 \\
\hline \multicolumn{7}{|l|}{ Women, $N=779$} \\
\hline Triglycerides (mg/dL) & $0.83(0.77-0.89)$ & $<0.001$ & $0.68(0.64-0.73)$ & $<0.001$ & $0.63(0.56-0.70)$ & $<0.001$ \\
\hline HDL cholesterol (mg/dL) & $0.25(0.19-0.32)$ & $<0.001$ & $0.38(0.29-0.39)$ & $<0.001$ & $0.28(0.22-0.34)$ & $<0.001$ \\
\hline LDL cholesterol (mg/dL) & $0.62(0.54-0.69)$ & 0.021 & $0.60(0.56-0.65)$ & $<0.001$ & $0.54(0.47-0.62)$ & 0.218 \\
\hline Non-HDL cholesterol (mg/dL) & $0.70(0.63-0.78)$ & 0.457 & $0.64(0.59-0.69)$ & $<0.001$ & $0.59(0.52-0.66)$ & 0.014 \\
\hline Total cholesterol/HDL cholesterol ratio & $0.78(0.72-0.84)$ & $<0.001$ & $0.69(0.65-0.74)$ & $<0.001$ & $0.70(0.63-0.76)$ & $<0.001$ \\
\hline Triglycerides/HDL cholesterol ratio & $0.84(0.78-0.89)$ & $<0.001$ & $0.70(0.65-0.74)$ & $<0.001$ & $0.69(0.62-0.75)$ & $<0.001$ \\
\hline LDL cholesterol/HDL cholesterol ratio & $0.74(0.68-0.81)$ & $<0.001$ & $0.68(0.63-0.72)$ & $<0.001$ & $0.68(0.62-0.75)$ & $<0.001$ \\
\hline
\end{tabular}

ROC, receiver operating characteristics; MetS, metabolic syndrome; HOMA-IR, homeostasis model assessment of insulin resistance; HMW, high molecular weight; AUC, area under ROC curve; $\mathrm{Cl}$, confidence interval; HDL, high-density lipoprotein; LDL, low-density lipoprotein. §HOMA-IR was calculated using the following formula; ffasting blood glucose $(\mathrm{mg} / \mathrm{dL}) \mathrm{X}$ immuno-reactive insulin $(\mathrm{mU} / \mathrm{mL})\} / 405$. Insulin resistance was defined as values exceeding the fourth quartile of HOMA-IR, and less than the first quartile of serum HMW adiponectin, respectively. 
they serve as valuable surrogates for insulin resistance in normoglycemic individuals [23], but this limitation is mitigated when the number of subjects evaluated is large, as in our study [24].Then, we used fasting insulin and the HOMA-IR as markers of insulin resistance. In addition, serum adiponectin is also considered to be an important modulator of insulin sensitivity [25,26] and dyslipidemia [27]. Thus, we define the cut off value originally according to the level of the top quintile of HOMA-IR distribution and first quartile of serum HMW adiponectin in our subjects.

The presence of hypertriglyceridemia, low HDL-C concentrations, and high TG/HDL-C ratios almost never occurred as isolated disorders, and were nearly always associated with insulin resistance because insulin affects TG and HDL-C metabolism [28]. Previous studies have shown that several lipid ratios have been proposed as clinically simple and useful indicators of hyperinsulinemia or insulin resistance. The TG/HDL-C ratio have shown similar potential for insulin resistance, though the generalizability of this association has been not entirely consistent. In 50 white Americans, both TG and TG/HDL-C ratio were acceptable markers for insulin resistance, with area under the ROC curve of 0.763 and 0.770 [29], respectively, and in a study in an East African population, the TG/HDL$\mathrm{C}$ ratio was found to be significantly associated with insulin resistance as measured by HOMA [30]. In contrast, recent studies have reported that theTG/HDL-C ratio was not significantly associated with insulin resistance in black adults [31] and adolescents [32]. The relationship between TG and TG/HDL-C with insulin resistance might be shown to differs by ethnicity. In our Japanese study, both TG/HDL-C and TC/HDL-C ratios as well as TG were useful makers of MetS-associated variables in both men and women, especially TG/HDL-C ratio in men. However, LDL-C was a weaker association with these variables. The present study provides additional information about lipid measures in both male and female Japanese.

Other lipid profiles have also shown their own relationship value for MetS. Kimm et al. [33] demonstrated that the lipid ratios of TC/HDL-C, LDL-C/HDL-C and TG/HDL-C, as well as TG and HDL-C, were each consistently associated with the number of metabolic syndrome components, insulin resistance quartiles (based on homeostatic model assessment), and log-transformed adiponectin quartiles. The lipids ratios that include information on at least two measures might have a more integrated explanation than single lipid measures such as TG or HDL-C [33]. In our study, the comparable results were seen.

Some limitations of this study must be considered. First, the response rate was as low as $35 \%$ that is usually the case in other conventional community studies in Japan. However, the relatively large sample size enabled the assessment of an extensive array of MetS-associated variables in relation to lipid profiles. Second, the crosssectional study design is limited in its ability to eliminate causal relationships between lipid profiles and MetS-associated variables. Third, our definition of HOMA-IR is based on a single assessment of BS and IRI, which may introduce misclassification bias. Fourth, we used $\mathrm{BMI} \geq 25 \mathrm{~kg} / \mathrm{m}^{2}$ to classify individuals with visceral obesity because waist circumference measurements were not available, which might have caused an under estimation of the effect of visceral obesity on MetS [34]. Therefore the demographics and referral source may limit generalizability.

In conclusion, the present study demonstrated that special lipid profiles are associated with MetS-associated variables in a general population. The ability to identify who have MetS could help health care professionals in bringing about lifestyle interventions. In that context, use of LDL-C/HDL-C ratio or TG/HDL-C ratio described in this report is simple and useful. Further prospective population-based studies are needed to investigate the changes in lipid metabolism by lifestyle interventions.

\section{Acknowledgements}

This work was supported in part by a grant-in-aid for Scientific Research from the Foundation for Development of Community (2009).

\section{Author details}

'Department of Community Medicine, Ehime University Graduate School of Medicine, Ehime 791-0295, Japan. ²Department of Geriatric Medicine, Ehime University Graduate School of Medicine, Ehime 791-0295, Japan.

${ }^{3}$ Department of Internal Medicine, Seiyo Municipal Nomura Hospital, Ehime 797-1212, Japan.

\section{Authors' contributions}

RK, YT, and KK participated in the design of the study, performed the statistical analysis and drafted the manuscript. NO, ToK, and TaK contributed to acquisition of data and its interpretation. ST and MA contributed to conception and design of the statistical analysis. TM conceived of the study, participated in its design, coordination and helped to draft the manuscript. All authors read and approved the manuscript.

\section{Competing interests}

The authors declare that they have no competing interests.

Received: 13 March 2011 Accepted: 17 May 2011

Published: 17 May 2011

\section{References}

1. Reaven GM: Role of insulin resistance in human disease. Diabetes 1988, 37(12):1595-1607.

2. DeFronzo RA, Ferrannini E: Insulin resistance. A multifaceted syndrome responsible for NIDDM, obesity, hypertension, dyslipidemia, and atherosclerotic cardiovascular disease. Diabetes Care 1991, 14(3):173-194.

3. Takeuchi H, Saitoh S, Takagi S, Ohnishi H, Ohhata J, Isobe T, Shimamoto K Metabolic syndrome and cardiac disease in Japanese men: applicability of the concept of metabolic syndrome defined by the National Cholesterol Education Program-Adult Treatment Panel III to Japanese men-the Tanno and Sobetsu Study. Hypertens Res 2005, 28(3):203-208. 
4. Shiwaku K, Nogi A, Kitajima K, Anuurad E, Enkhmaa B, Yamasaki M, Kim JM, Kim IS, Lee SK, Oyunsuren T, Yamane Y: Prevalence of the metabolic syndrome using the modified ATP III definitions for workers in Japan, Korea and Mongolia. J Occup Health 2005, 47(2):126-135.

5. Galassi A, Reynolds K, He J: Metabolic syndrome and risk of cardiovascular disease: a meta-analysis. Am J Med 2006, 119(10):812-819

6. Ford ES: Risks for all-cause mortality, cardiovascular disease, and diabetes associated with the metabolic syndrome: a summary of the evidence. Diabetes Care 2005, 28(7):1769-1778.

7. Ziemke F, Mantzoros CS: Adiponectin in insulin resistance: lessons from translational research. Am J Clin Nutr 2010, 91(1):258S-261S.

8. Seino $Y$, Hirose $H$, Saito I, Itoh H: High-molecular-weight adiponectin is a predictor of progression to metabolic syndrome: a population-base 6year follow-up study in Japanese men. Metabolism 2009, 58(3):355-360.

9. Pischon T, Girman CJ, Hotamisligil GS, Rifai N, Hu FB, Rimm EB: Plasma adiponectin levels and risk of myocardial infarction in men. JAMA 2004, 291(14):1730-1737.

10. Lara-Castro C, Luo N, Wallace P, Klein RL, Garvey WT: Adiponectin multimeric complexes and the metabolic syndrome trait cluster. Diabetes 2006, 55(1):249-259.

11. Expert Panel on Detection, Evaluation, and Treatment of High Blood Cholesterol in Adults: Summary of the second report of the National Cholesterol Education Program (NCEP) Expert Panel on Detection, Evaluation, and Treatment of High Blood Cholesterol in Adults (Adult Treatment Panel II). JAMA 1993, 269(23):3015-3023.

12. Reaven GM: Insulin resistance, the insulin resistance syndrome, and cardiovascular disease. Panminerva Med 2005, 47(4):201-210.

13. Kimm H, Lee SW, Lee HS, Shim KW, Cho CY, Yun JE, Jee SH: Associations between lipid measures and metabolic syndrome, insulin resistance and adiponectin. - Usefulness of lipid ratios in Korean men and women -. Circ J 2010, 74(5):931-937.

14. McLaughlin T, Reaven G, Abbasi F, Lamendola C, Saad M, Waters D, Simon J, Krauss RM: Is there a simple way to identify insulin-resistant individuals at increased risk of cardiovascular disease? Am J Cardiol 2005, 96(3):399-404.

15. McLaughlin T, Abbasi F, Cheal K, Chu J, Lamendola C, Reaven G: Use of metabolic markers to identify overweight individuals who are insulin resistant. Ann Intern Med 2003, 139(10):802-809.

16. Laws A, Reaven GM: Evidence for an independent relationship between insulin resistance and fasting plasma HDL-cholesterol, triglyceride and insulin concentrations. J Intern Med 1992, 231(1):25-30.

17. Friedewald WT, Levy RI, Fredrickson DS: Estimation of the concentration of low-density lipoprotein cholesterol in plasma, without use of the preparative ultracentrifuge. Clin Chem 1972, 18(6):499-502.

18. Matthews DR, Hosker JP, Rudenski AS, Naylor BA, Treacher DF, Turner RC: Homeostasis model assessment: insulin resistance and beta-cell function from fasting plasma glucose and insulin concentrations in man. Diabetologia 1985, 28(7):412-419.

19. Examination committee of criteria for diagnosis of metabolic syndrome in Japan: Definition and criteria for the diagnosis of metabolic syndrome. J Jpn Soc Int Med 2005, 94(4):794-809.

20. The examination Committee of Criteria for "Obesity Disease" in Japan. Japan Society for Study of Obesity: New criteria for 'obesity disease' in Japan. Circ J 2002, 66(11):987-992.

21. Yeni-Komshian H, Carantoni M, Abbasi F, Reaven GM: Relationship between several surrogate estimates of insulin resistance and quantification of insulin-mediated glucose disposal in 490 healthy nondiabetic volunteers. Diabetes Care 2000, 23(2):171-175.

22. Rutter MK, Meigs JB, Sullivan LM, D'Agostino RB Sr, Wilson PW: Insulin Resistance, the Metabolic Syndrome, and Incident Cardiovascular Events in the Framingham Offspring Study. Diabetes 2005, 54(11):3252-3257.

23. Laakso M: How good a marker is insulin level for insulin resistance? Am J Epidemiol 1993, 137(9):959-965.

24. Bonora E, Kiechl S, Willeit J, Oberhollenzer F, Egger G, Targher G, Alberiche M, Bonadonna RC, Muggeo M: Prevalence of insulin resistance in metabolic disorders: the Bruneck Study. Diabetes 1998, 47(10):1643-1649.

25. El Feghali R, Topouchian J, Pannier B, Asmar R: Ageing and blood pressure modulate the relationship between metabolic syndrome and aortic stiffness in never-treated essential hypertensive patients. A comparative study. Diabetes Metab 2007, 33(3):183-188.
26. Weyer C, Funahashi T, Tanaka S, Hotta K, Matsuzawa Y, Pratley RE, Tataranni PA: Hypoadiponectinemia in obesity and type 2 diabetes: close association with insulin resistance and hyperinsulinemia. J Clin Endocrinol Metab 2001, 86(5):1930-1935.

27. Matsubara M, Maruoka S, Katayose S: Decreased plasma adiponectin concentrations in women with dyslipidemia. J Clin Endocrinol Metab 2002, 87(6):2764-2769.

28. Lewis GF, Uffelman KD, Szeto LW, Steiner G: Effects of acute hyperinsulinemia on VLDL triglyceride and VLDL apoB production in normal weight and obese individuals. Diabetes 1993, 42(6):833-842.

29. Kim-Dorner SJ, Deuster PA, Zeno SA, Remaley AT, Poth M: Should triglycerides and the triglycerides to high-density lipoprotein cholesterol ratio be used as surrogates for insulin resistance? Metabolism 2010, 59(2):299-304.

30. Bovet $P$, Faeh $D$, Gabriel A, Tappy L: The prediction of insulin resistance with serum triglyceride and high-density lipoprotein cholesterol levels in an East African population. Arch Intern Med 2006, 166(11):1236-1237.

31. Sumner $A E$, Finley KB, Genovese DJ, Criqui MH, Boston RC: Fasting triglyceride and the triglyceride-HDL cholesterol ratio are not markers of insulin resistance in African Americans. Arch Intern Med 2005, 165(12):1395-1400

32. Hoffman RP: Increased fasting triglyceride levels are associated with hepatic insulin resistance in Caucasian but not African-American adolescents. Diabetes Care 2006, 29(6):1402-1404.

33. Kimm H, Lee SW, Lee HS, Shim KW, Cho CY, Yun JE, Jee SH: Associations between lipid measures and metabolic syndrome, insulin resistance and adiponectin. - Usefulness of lipid ratios in Korean men and women Circ J 2010, 74(5):931-937.

34. Kawamoto R, Ohtsuka N, Ninomiya D, Nakamura S: Carotid atherosclerosis in normal-weight metabolic syndrome. Intern Med 2007, 46(21):1771-1777.

\section{doi:10.1186/1476-511X-10-79}

Cite this article as: Kawamoto et al:: Relationships between lipid profiles and metabolic syndrome, insulin resistance and serum high molecular adiponectin in Japanese community-dwelling adults. Lipids in Health and Disease 2011 10:79.

\section{Submit your next manuscript to BioMed Central and take full advantage of:}

- Convenient online submission

- Thorough peer review

- No space constraints or color figure charges

- Immediate publication on acceptance

- Inclusion in PubMed, CAS, Scopus and Google Scholar

- Research which is freely available for redistribution

Submit your manuscript at www.biomedcentral.com/submit
C Biomed Central 